\title{
NUTRIGENÉTICA APLICADA: DIETA PERSONALIZADA Y FORMACIÓN ACADÉMICA PARA LA PRÁCTICA PROFESIONAL
}

\section{NUTRIGENETICS IN PRACTICE: PERSONALIZED DIET AND ACADEMIC TRAINING FOR PROFESSIONAL ADVICE}

\author{
Marta Coronado H., Salvador Vega y León, Rey Gutiérrez T., \\ José Pérez G., Karina Peláez M. \\ Departamento de Producción Agrícola y Animal \\ Universidad Autónoma Metropolitana - Xochimilco, México.
}

\begin{abstract}
The aim of this paper is to focus on the new perspectives which are offered by the advent of genomics (nutrigenetic) and their potential on individual nutrition recommendations. Moreover, this article examines the influence of polymorphism on the response to dietary patterns which is the key player in the nutrigenetic field. It is clear that genetic variation influences nutritional requirements, therefore it will influence personalized nutrition recommendation. This paper also focuses on consumer population perspectives concerning nutrigenetics and examines the academic training of future health professionals.
\end{abstract}

Key words: nutrigenetic; polymorphism; personalized diet; consumer population; professional training.

Este trabajo fue recibido el 1 de Marzo de 2011 y aceptado para ser publicado el 11 de Septiembre de 2011.

\section{INTRODUCCIÓN}

El objetivo de este artículo es comunicar los avances de la nutrigenética, la cual plantea un nuevo paradigma alimentario, al sugerir la posibilidad de individualizar la alimentación de acuerdo con los componentes genéticos de cada individuo. Se percibe que en los próximos 25 años habrá una nueva revolución tecnológica que incluirá, entre otras, a la biotecnología, la nanotecnología y a la genómica (1). En consecuencia, surgirá un cúmulo de información genética y nuevas técnicas de alta resolución que permitirán una cantidad relevante de herramientas para los estudios en el campo que asocia genes-nutrición. La bioinformática, la biología celular y molecular y la bioquímica, también formarán parte de esta nueva era tecnológica e incidirán en los nuevos diseños curriculares en las instituciones educativas.

Este trabajo incluye algunos aportes de estudios poblacionales en México (Distrito Federal) y en Estados Unidos en donde la población consumidora plantea su posición en torno a un posible cambio de dieta, si su mapa genético sugiere el cuidado de la salud por esta vía. Además, se aborda la formación académica de los profesionales de la salud que estarán involucrados en la aplicación práctica de la nutrigenética.

Sin duda, la temática anterior representa un nicho de investigación importante y atractivo para diversos grupos científicos y para el desarrollo e innovación, de insumos génicos y de nuevos productos alimenticios (2).

\section{NUTRIGENÓMICA Y NUTRIGENÉTICA}

La nutrigenómica estudia los mecanismos por los cuales los nutrientes actúan como señales químicas para incidir en la expresión de los genes, y de esta forma modificar la síntesis de proteínas y el funcionamiento de las diversas rutas metabólicas. La nutrigenética analiza la respuesta de diferentes genotipos a la ingesta de los nutrientes y la forma en que esta relación determina la susceptibilidad de un sujeto a padecer una enfermedad particular (3).

La nutrigenética implica el estudio de los polimorfismos individuales y la literatura advierte sobre la analogía con la farmacogenómica que busca fármacos propios para cada paciente y su padecimiento (4).

Esta rama del conocimiento científico que considera las variaciones genéticas individuales lleva a la siguiente propuesta: DETERMINADO NUTRIENTE 
$=$ DETERMINADO INDIVIDUO. Se puede inferir que este principio sería la contraparte de la nutrición basada en recomendaciones poblacionales, que plantea una ingesta de nutrientes a partir de estudios epidemiológicos y contenidas en las Recommended Dietary Allowances (RDAs por sus siglas en inglés). A partir de esas recomendaciones se sugiere un nivel medio diario de consumo de un nutriente suficiente, para cubrir los requerimientos nutricionales del $97 \%-98 \%$ de una población sana de edad específica (5).

Sin embargo, la nutrigenética podrá en un futuro, identificar subgrupos de población que sean menos eficientes en manejar la ingesta de alimentos y las vías metabólicas específicas para éstos de tal forma que se puedan sugerir recomendaciones de nutrientes acordes con su perfil genético (6). La figura 1 da cuenta de estas relaciones para lograr la aplicación de la nutrigenética a la salud de la población.

\section{POLIMORFISMOS Y NUTRIGENÉTICA}

El estudio de las variaciones genéticas entre individuos y su respuesta a nutrientes particulares, aunque consuman la misma dieta, ha sido motivo de análisis en tanto ocurre por efecto de los polimorfismos. Éstos implican la diferencia en la secuencia de bases del DNA (variación presente en por lo menos 1\% de la población). El más común es el de un sólo nucleótido (SNPs, por sus siglas en inglés que significan single nucleotide polymorphism), $(6,7)$.

Los polimorfismos pueden surgir por una mutación que involucra el cambio en la base de un nucleótido hasta variantes que involucran centenares de bases, sea por pérdida o inserción. Este fenómeno ocurre con bastante frecuencia en rangos que se observan en uno de cada 1000, a uno en cada 100-300 bases. Por lo anterior se plantea que el genoma humano contiene alrededor de diez millones de SNPs (8). Cabe advertir, que las mutaciones en general se perciben como anormales o patológicas, sin embargo, los SNPs se consideran normales entre los individuos y se podría apuntar que son fenómenos silentes (7).

Es importante advertir que la detección o el análisis de todos los SNPs de un sujeto, en este momento una tarea difícil, pero la naturaleza aporta una ventaja, por ello se ha podido observar que los polimorfismos cercanos en la secuencia del DNA de un gen, se heredan juntos (haplotipo) y que la mayor parte de las regiones cromosómicas sólo contiene algunos de estos haplotipos comunes, lo cual facilita su estudio (5).

Sólo como ejemplo de los polimorfismos más estudiados, se puede apuntar el que ha propiciado una recomendación nutricia y está relacionado con el ácido fólico. Este nutriente tiene en la actualidad una amplia campaña para su consumo, en particular para las mujeres embarazadas. Se ha estudiado (9) esta relación en la región sub-sahariana de África en donde se ha detectado una baja presencia del polimorfismo asociado, en tanto aumenta en sujetos caucásicos y presenta alta frecuencia en italianos e hispanos, como se ha observado en México.

El polimorfismo asociado es el C677T que presenta un cambio en las bases moleculares de citosina a timina, lo cual genera otro cambio pero en la estructura enzimá-

\section{FIGURA 1}

\section{Nutrigenética para la salud de la población.}

A.

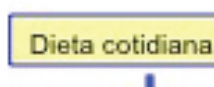

B.

\begin{tabular}{|c|c|c|}
$\begin{array}{c}\text { Genética del metabolismo } \\
\text { del nutriente }\end{array}$ & $+\begin{array}{c}\text { Genética del } \\
\text { receptor del } \\
\text { nutriente }\end{array}$
\end{tabular}$\quad+\begin{gathered}\text { Heterogeneidad } \\
\text { de la respuesta } \\
\text { individual al nutriente }\end{gathered}$

C.

Proceso de: absorción metabolismo excreción
Susceptibilidad a determinada enfermedad
Dieta personalizada; prevención y tratamiento 
tica, particularmente de alanina por valina (A222V). Lo anterior da lugar a que la enzima participante, MTHFR (metilentetrahidrofolato reductasa) sea una enzima más termolábil y con una disminución (50\%) en su actividad. Se ha observado que esta variante específica termolábil, propicia una deficiencia parcial en un 5 a $30 \%$ de la población normal (3).

Se ha reconocido una forma homocigótica (TT) y otra heterocigótica (CT), lo cual implica susceptibilidad para los sujetos que portan estas formas génicas, las cuales se asocian con el consumo de algunos nutrientes como folatos, vitaminas B12 (cianocobalamina), y B6 (piridoxina) (3).

El C677T propicia la reducción significativa de las concentraciones de folato en los glóbulos rojos y en el plasma de mujeres en general, y en mujeres embarazadas (TT homocigotos) se observa una disminución mayor de estos niveles de folato. El problema se asocia con una reducción en la actividad enzimática y el incremento del amino ácido homocisteína en uno de los pasos metabólicos, lo cual puede conducir a defectos de los tubos neurales y problemas cardiovasculares (10). Una alta concentración de la homocisteína tiene un efecto tóxico sobre el endotelio de las arterias y puede causar lesión aterosclerótica (11).

En un estudio (12) con mujeres sanas, el $8.3 \%$ de embarazadas y el $12.9 \%$ de no embarazadas fueron positivas al C677T y presentaron niveles de folatos en eritrocitos, significativamente menores a los sujetos sin este polimorfismo. Un 5\% a 15\% de la población general son homocigotos para el polimorfismo, mencionado, por lo que se recomienda un aumento de folato en la dieta diaria (12).

Las enzimas involucradas son la metionina sintetasa que convierte la homocisteína a metionina y la metilentetrahidrofolato reductasa (MTHFR) mencionada antes, que propicia la conversión del 5,10metilentetrahidrofolato a 5-metilentetrahidrofolato. En este proceso se requiere vitamina B12 que actuará en el paso de remetilación de homocisteína a metionina, como se observa en la figura 2.

\section{DIETA DIARIA Y MODULACIÓN GENÉTICA INDIVIDUAL}

Hay evidencia que ratas alimentadas con proteína de soya disminuyen la expresión del factor de transcripción

\section{FIGURA 2}

\section{Efecto del polimorfismo C677T en la vía metabólica del ácido fólico.}

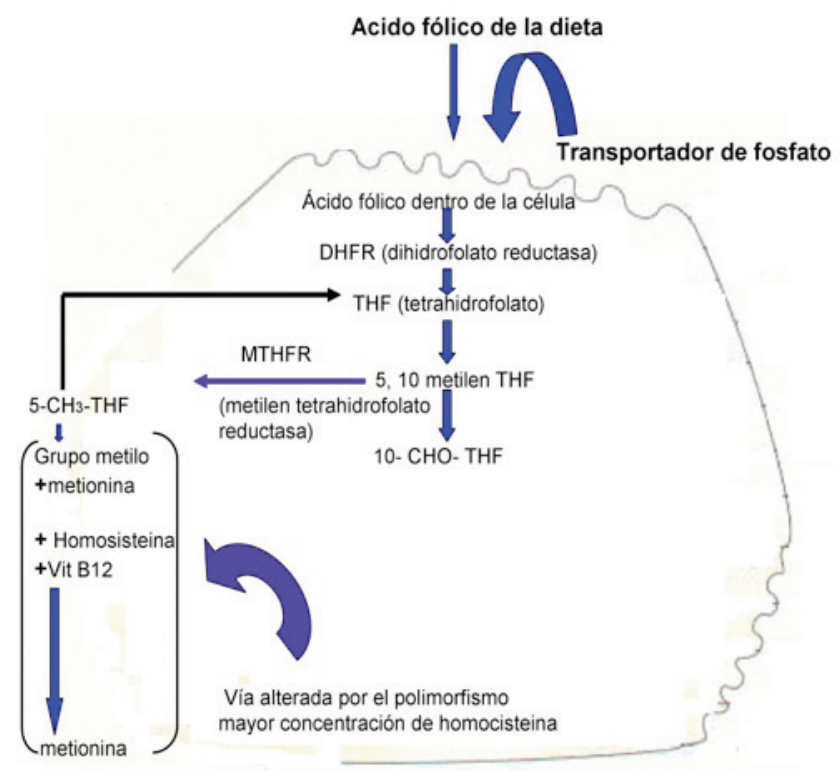

Fuente: Modificado de Silveira, Martínez, Carrazo, 2007; Meshkin, Blum, 2007. 
SREBP-1 y que después de una hora de consumo de dieta con caseína (como fuente proteica), aumentó 3.5 veces la concentración de insulina sérica (respecto al nivel basal) en tanto la ingesta de proteína de soya sólo elevó estos niveles en un 50\%.

Por otra parte, la ingestión de una dieta con alto contenido de isoflavonas provenientes de proteína de soya, mejoran la insulinorresistencia en ratas Zucker obesas y que parte de estos efectos benéficos se asocian a otra familia de factores de transcripción, como son los PPARs (14).

Los resultados indican que la ingestión a corto o largo plazo de proteína de soya mantiene normal los niveles de insulina sérica comparados con animales alimentados con caseína. También se ha observado que en el hígado, se genera una baja expresión del RNAm de SREBP-1 y en consecuencia baja la biosíntesis de las enzimas lipogénicas, las sintetasas de ácidos grasos y la enzima málica, todas involucradas en el metabolismo lipogénico. Lo contrario se observó en dietas con caseína (14 - 17).

El arroz por su contenido de inositol hexafosfato, puede inhibir la transformación celular inducida por el factor de crecimiento celular, al incidir en la enzima fosfatidilinositol 3-cinasa. Otro compuesto presente en alimentos como uvas o el vino, es el resveratrol, puede afectar las rutas de señalización celular (18).

El bajo consumo de frutas, hortalizas, vitamina $\mathrm{C}$ y alfa-tocoferol se ha relacionado con el riesgo del cáncer de mama en mujeres, si ellas son portadoras de un polimorfismo en la enzima superóxido dismutasa, dependiente de un micronutriente, el manganeso. La deficiencia de otros micronutrientes como el ácido fólico, las vitaminas B12, B6, C y E, niacina, y los minerales hierro y zinc, pueden alterar el DNA con un efecto parecido al de las radiaciones, que causa roturas a la doble cadena, lesiones oxidativas o ambas. Lo anterior sugiere un riesgo para el desarrollo de cáncer humano (19).

En el caso de la lactosa (azúcar de la leche), se plantea (4) que una mutación ocurrida hace unos 9,000 años, en la población del norte de Europa dio lugar a la expresión del gen de la lactasa (LCH locus). Al respecto se han detectado 11 polimorfismos en cuatro haploides (A,B,C,U) y se ha observado también que el polimorfismo C1391OT ubicado por encima del LCH se asocia con la tolerancia a la lactosa. En el caso del haplotipo A, que permite la tolerancia al nutriente en cuestión, está presente en un $86 \%$ de la población europea del norte, pero disminuye a un $36 \%$ en la parte sur de la misma región (3), lo que implica variación en diversas regiones del mundo, como es el caso del continente americano, en particular México.
Así, la variación genética CT se vincula con la persistencia o no de la lactasa, en tanto el genotipo CC se relaciona con la no persistencia de la enzima (20).

Una de las soluciones proviene de la industria alimentaria con el desarrollo y venta de leches sin lactosa, que incluso en varios países tienen una oferta y consumo amplio.

Otro alimento estudiado es el salvado de avena y su relación con el genotipo APOE3/3 que puede inducir una respuesta hipocolesterolémica a las cuatro semanas de ingesta del alimento. Sin embargo, los sujetos con APOE4/4 o APOE4/3 no presentan cambios (12).

La complejidad de la dieta diaria se puede ejemplificar con los alimentos que se ingieren en una comida sencilla. Por ejemplo al incluir el aceite de oliva, se generan cientos de componentes como son diferentes ácidos grasos, triacilglicéridos, esteroles, ésteres de esterol y tocoferoles, los cuales van a regiones celulares diversas durante el metabolismo. Lo anterior es sólo un ejemplo simple de la relación entre la dieta ingerida y su participación en la salud del individuo a lo cual, en la actualidad, se agregarían las posibles alteraciones genéticas que los alimentos pueden producir de manera directa o indirecta.

\section{PERSPECTIVAS DE LA POBLACIÓN CONSUMIDORA SOBRE ESTE NUEVO PARADIGMA ALIMENTARIO}

Es importante revisar la respuesta de la población hacia las nuevas tecnologías alimentarias y los nuevos alimentos que encontrará en los anaqueles de su expendio comercial y recordar que la comida es un elemento cultural, en el que intervienen creencias personales, sean religiosas o no, un estilo de vida o vigilancia particular de la salud.

Por ello, los consumidores, los profesionales de la salud, diseñadores de políticas públicas, académicos o empresarios deben opinar antes de que los productos se oferten en el mercado (21), porque en la aceptación de un nuevo producto, la potencial población consumidora tiene la última palabra.

En este entorno la empresa alimentaria no quiere perder el "tren de los alimentos nutrigenómicos" (7) y diversifica su cartera de productos en un momento en el que se enfrentará a un crecimiento exponencial de la oferta-demanda, con un posible aumento de la fortificación y de los alimentos funcionales (7\%), sobre todo por el conocimiento que obtendrá la población sobre los mecanismos de acción de los componentes bioactivos de los alimentos (22). Incluso en un informe del Círculo de Innovación en Biotecnología de Madrid se incluyen 23 empresas (Sciona, Genelex, Genetic Health, Nutra- 
metrix, WellGen, Celera, entre otras) con servicios o productos nutrigenómicos (7).

La recién creada NuGO (European Nutrigenomics Organization) se ha dado a la tarea de llevar a la práctica los datos de la nutrición molecular, con el objetivo de hacer predicciones precisas de los efectos, sean benéficos o no, de los componentes dietarios sobre todo para la prevención de enfermedades crónicas como las cardiovasculares o la diabetes, entre otras (23). Además, la European Food Safety Authority EFSA o la Food and Drugs Administration (FDA) de los Estados Unidos, han establecido un marco legislativo para las declaraciones de salud y nutrición en los alimentos (23).

Estos esfuerzos de información a la población consumidora han propiciado que en Estados Unidos la Fundación del Consejo Internacional de Información Alimentaria (IFIC) cuya función es proporcionar información sobre seguridad alimentaria y nutrición, haya realizado diversos estudios $(1998,2000,2002,2005)$ sobre genómica y nutrición, en población abierta. En la encuesta de 2005 (con 1012 adultos) se exploró el ámbito de la nutrición personalizada (24).

Los datos relevantes mostraron que el $70 \%$ de la población encuestada estaba interesada en aprender más sobre el concepto y el uso de la nutrigenética para programar sus recomendaciones nutrimentales y optimizar su salud.

El 90\% manifestó que los antecedentes familiares (genética) tienen una relación importante en la salud y un $71 \%$ estaría de acuerdo con utilizar su información genética para programar recomendaciones dietarias. Alrededor del $70 \%$ de los encuestados prefirió "nutrición personalizada", $68 \%$ prefirió "nutrición individualizada", los términos más técnicos como nutrigenómica o nutrigenética no resultaron con alta preferencia. Algunos encuestados plantearon sus dudas respecto al tema, sobre todo por la confidencialidad y la privacidad que todavía no saben cómo se mantendrá para aquellos que se realizaran un perfil genético (24).

Cabe advertir que la Fundación IFIC comunica sus resultados a un amplio auditorio como profesionales de la salud, educadores, periodistas, funcionarios gubernamentales y otros que proporcionan información a la población consumidora, aparte de contar con el apoyo de las industrias alimentaria y agrícola (25). En 2005, los datos de IFIC se llevaron a la Conferencia Internacional sobre Nutrigenómica-Oportunidades en Asia y se planteó la diversidad étnica y la adaptación genética en tiempos de migración, como son los actuales, y su relación con los patrones dietarios. Se analizaron una diversidad de puntos, entre otros: genes-nutrimentos y salud, nutrición, epidemiología molecular y enfermedades, genómica y suministro de alimentos, población individual y futuro de la nutrigenética.

La agencia Cogent Research, aplica una encuesta anual de tendencias sobre las actitudes que presentan los consumidores norteamericanos hacia la información genética individual y su relación con la salud. Se señala que la mitad de los encuestados "están preparados" para consumir alimentos acordes con su perfil genético y se favorecen las recomendaciones dietarias individuales, más que los regímenes generales. Además se prefiere el término "nutrición personalizada" en lugar de nutrigenética y de igual manera, similar a los datos de IFIC, el $71 \%$, considera que la genética tiene un papel fundamental en la salud durante toda la vida de una persona.

El 73\%, en este estudio (25), expresó preocupación por el uso de la información genética, sea por la aplicación o custodia de la misma, temor que también se percibe en otros grupos de población.

\section{ESTUDIO LOCAL EXPLORATORIO ENTRE POBLACIÓN CONSUMIDORA}

Con el objetivo de obtener información local sobre la aceptación o rechazo de la nutrigenética, se realizó una encuesta exploratoria, en la Ciudad de México (2010) en un grupo de pacientes (160) de clínicas de salud. Los resultados indicaron que la aceptación hacia la importancia de realizarse un mapeo genético personal fue alta $(72.25 \%)$ y el nivel de acuerdo para cambiar su dieta actual por una personalizada, como producto de su perfil genético, fue de $71.3 \%$. Estos datos coinciden con los de IFIC (24), en el sentido de una preocupación actual por la salud y la posibilidad de que la nutrigenética se explore con más estudios y mayor precisión. De hecho no hay un rechazo a priori de esta nueva aportación para valorar la salud y la nutrición.

Se realizaron, 17 entrevistas a especialistas (médicos, nutriólogos y psicólogos) quienes plantearon que una dieta más saludable será aquella que se adapte al perfil genético de cada persona y que las dietas personalizadas mejorarían la calidad de vida e impedirían o atrasarían el desarrollo de algunas enfermedades.

Sin embargo, es posible que para la mayoría de la población no sea fácil modificar su hábito dietario, además no deseen afrontar la idea de desarrollar una enfermedad y enterarse de ello por el mapeo genético. Se reconoce que en la actualidad todavía se tiene poco conocimiento al respecto y casi ninguna accesibilidad a estos estudios y tratamientos.

Otro aporte señala que la población no tiene una cultura de alimentación sana, sobre todo en los niños que tienen preferencia por productos procesados poco nutritivos. El arraigo ancestral a un tipo de alimentos 
o platillos, hace difícil el cambio a corto plazo, aunque no imposible con una educación alimentaria desde la infancia.

Una propuesta es buscar opciones alimentarias que suplan algún alimento que haya que eliminar de la dieta. En la actualidad se exploran estas alternativas y la industria alimentaria participa y tiene ofertas en el mercado.

Un punto relevante que abordaron los entrevistados es el grupo de población indígena, cuya alimentación en el campo está basada en la siembra de autoconsumo, y su dieta se elabora con esta producción, lo cual difícilmente podrá cambiar. En México, como en otros países no hay las condiciones ni el nivel de vida que permita "echar por la borda" las costumbres alimentarias, lo cual habrá de considerarse si la nutrigenética fuese la vía para elaborar las recomendaciones nutricionales en los programas de salud pública.

\section{EDUCAR PARA EL FUTURO DE LA NUTRIGENÉTICA: PROFESIONALES} DE LA SALUD

Los conocimientos profesionales de las futuros trabajadores de la salud tendrán un perfil diferente al actual, con la inclusión no sólo de la genética molecular humana, individual y poblacional además de las áreas clásicas sobre biología (anatomía, fisiología microbiología, nutrición) entre otras.

Un ejemplo práctico (26), plantea lo siguiente. Una señora de unos 50 años, acude a una consulta médica con antecedentes familiares de enfermedades cardiovasculares. La idea de la paciente es obtener indicaciones para llevar una dieta y vida diaria saludables, que permitan en lo posible disminuir el riesgo de la enfermedad. El especialista revisa el expediente electrónico del genoma para observar el perfil genético de la paciente, y agrega datos sobre la salud de los familiares (padre y madre), los medicamentos y la dieta que han llevado. Se registra la dieta actual de la paciente, la práctica de ejercicio físico y se analiza si estas prácticas son adecuadas para sus características personales.

El perfil genético indica un polimorfismo en el gene APOA1, asociado con bajos niveles de HDL colesterol, cuando el consumo de ácidos grasos poliinsaturados es bajo. El genoma también muestra un polimorfismo en el gene de la lipasa hepática, el cual es benéfico porque aumenta los niveles de HDL-C cuando la ingesta de lípidos es menor al 30\% de la energía total.

Después de analizar los datos se extienden las recomendaciones para que la persona aumente su consumo de estos ácidos grasos poliinsaturados, mientras mantiene una baja ingesta de grasas animales. Además se le dan recomendaciones de estilo de vida saludable (ejercicio, eliminación del tabaquismo, baja de estrés, entre otros) acordes con su genotipo. Se le explica a la paciente que sólo los cambios en su estilo de vida, que incluye la dieta, serán la opción para controlar su perfil lipídico aterogénico.

Toda la información se registra en una bitácora electrónica personal de la paciente y se programan nuevas citas con el consejo de registrar los datos que surjan y así el nutriólogo pueda contar con datos nuevos en cada consulta.

Lo anterior plantea que en este nuevo entorno clínico, un invaluable apoyo adicional será la investigación de laboratorio o de campo con las herramientas que estas práctica conllevan como la estadística (muestreos, manejo de variables, análisis de datos, ente otros), además de tomar en cuenta otros aspectos que no por cotidianos son menos importantes para efectos investigativos: ¿cómo controlar la ingesta alimentaria de una persona?, sobre todo si se quiere trabajar con un número amplio de sujetos. El tamaño de la muestra que puede ser pequeño, errores en la selección de los grupos control o en la estratificación de la población incluso datos sobreinterpretados, que plantean dificultades sobre la confiabilidad de los resultados obtenidos.

Algunos estudios de campo como el realizado entre profesionales de la salud (unos 65000 afiliados en la American Dietetic Association) publica una revisión sobre genómica nutricional y apunta que hay un número limitado de consejeros certificados sobre genética entre los miembros de esta asociación (27). En otro estudio (28) el cual incluyó 913 sujetos, los encuestados respondieron, con mayor frecuencia, que entre los padecimientos relacionados con un perfil genético que requiere de cambios en la dieta y estilo de vida se incluyen los cardiovasculares (87\%), sensibilidad a la insulina (78\%) y salud ósea (66\%). Respecto a los beneficios de aplicar la nutrigenética se consideró que ésta influirá para recomendar una dieta individualizada $(84 \%)$ y será una vía para fundamentar las recomendaciones nutricias (76\%). También podrá apoyar la prescripción dietética para el manejo o prevención de ciertos padecimientos (75\%).

Entre las barreras más importantes se señalaron, la falta de educación continua (73\%) o la falta de conocimientos previos de los profesionales de la salud para sustentar la práctica de la nutrigenética $(81 \%)$, además de la falta de expertos para validar la experiencia profesional en este campo (72\%).

En este contexto educativo también se ha diseñado un programa multimedia, para estudiantes y público en general, denominado The Human Genome Project: Exploring Our Molecular Selves, el cual puede consultarse en línea (29). 
Otro aporte similar de corte académico, es el curso digital interactivo (30) que está dirigido a estudiantes universitarios de diversas disciplinas afines (biotecnología, nutrición, medicina, entre otros), que desean participar en un curso introductorio sobre nutrición molecular.

Para apuntalar la calidad de este curso un grupo de expertos de NuGo consideró que puede impartirse a estudiantes de licenciatura, maestría o doctorado (30), lo cual recomienda su análisis y en su caso la aplicación del mismo.

\section{ESTUDIO LOCAL EXPLORATORIO ENTRE FUTUROS PROFESIONALES DE LA SALUD}

Respecto al estudio de la población consumidora, se obtuvo información comparativa local, entre los futuros profesionales de la salud. Se realizó un estudio exploratorio (encuesta con 240 sujetos) en la Ciudad de México (2010), con estudiantes universitarios que cursaban la última fase de sus licenciaturas y se formaban como profesionales de la salud (medicina y nutrición). Los resultados fueron similares a los de otros autores (28). En principio los alumnos consideraron $(57.5 \%)$ que hay influencia de los nutrientes en la actividad genética humana. Aportaron su calificación positiva (73.2\% $-84.5 \%$ ) a una batería de reactivos (invertir en salud, conocer el riesgo de enfermedades genéticas, mejorar la calidad de vida, diseñar una dieta de acuerdo con el perfil genómico) que evaluó los beneficios de realizarse un mapeo genético personal. Otro punto valorado fueron los beneficios de la nutrigenética para la población mexicana. En esta pregunta también se trabajó con una batería de reactivos (aplicación de una dieta de acuerdo con la predisposición genética, prevenir enfermedades, curar enfermedades) y la respuesta fue positiva de $73.8 \%$ - 86.4\%. Respecto a los medios para informarse sobre la nutrigenética (también con una batería de reactivos, las respuestas se ubicaron entre congresos y revistas especializadas (69.1\%) y entre revistas e Internet (61.3\% - $82.3 \%$ ). Otros reactivos como cursos académicos o conferencias fueron de menor elección. Finalmente 55\% consideró que la nutrigenética tendrá, en algunas décadas un desarrollo entre bueno y excelente.

Los datos anteriores confirman, igual que otros autores $(26,28)$, que los profesionales de la salud que se dediquen a la práctica de la nutrigenética, requieren de una base sólida de conocimientos, no sólo sobre alimentos y nutrición, sino de ciencia básica y genética.

Como corolario de este apartado surge la reflexión sobre el papel fundamental que tendrán las universidades frente a este nuevo paradigma, en tanto deberán integrar en sus curriculos las herramientas y contenidos académicos necesarios a nivel licenciatura o en los posgrados y propiciar la actualización constante.

Un aporte final que los autores consideran relevante en este texto, para apoyar el punto sobre educación, es recomendar algunos títulos que sin duda permiten ampliar el conocimiento sobre la nutrigenética. (Ver bibliografía: 26 y 30-34).

\section{CONCLUSIONES}

En la actualidad se percibe un proceso de cambio respecto al concepto de alimentación ideal. Antes se sugerían recomendaciones nutricionales para grandes grupos de población susceptibles o no a determinado padecimiento. Lo anterior plantea un nuevo paradigma para los programas nutricionales de salud pública, porque se podrían continuar con aquellas recomendaciones de nutrientes para toda la población o sugerir dietas personalizadas, como lo establece la nutrigenética.

Sin embargo, la complejidad de este extenso ámbito de la nutrigenética tomará tiempo entre ciencia básica y aplicaciones tecnológicas, por lo que la investigación, el desarrollo y la innovación requerirán una gama diversa de estudios experimentales y demoscópicos y avalar la calidad y validez clínica de los marcadores nutrigenéticos específicos.

Los autores de este artículo, consideran que la nutrición personalizada está próxima (unos 10 años) de considerarse como propuesta de salud, en diversas regiones del mundo, como lo plantea la mayor parte de la literatura.

Desde la perspectiva científica se puede calificar esta disciplina como nutrigenética, o utilizar un término como nutrición o dieta personalizada, dieta individual o individualizada, de tal forma que sea comprensible para la población en general y se puedan apoyar acciones de salud pública.

Un perfil génico que predisponga a una enfermedad requerirá de absoluta discresión para el interesado, por su repercusión inmediata o futura en su desempeño laboral o en la contratación de un seguro personal.

Por lo anterior algunas interrogantes son pertinentes: ¿un individuo de manera personal deberá recurrir a su examen genómico o requerirá del apoyo de un profesional en nutrición?, ¿el estudio deberá ser compartido con la familia, en el ámbito laboral o ser de estricta reserva personal?, ¿el alcance de los estudios deberá incluir a toda la población y considerar precios accesibles?

En el ámbito socio-económico la relación tecnocientífca de la nutrigenética es innegable y de ella se derivarán diversos nichos de investigación, desarrollo e innovación tanto en empresas de insumos génicos, como en las de alimentos. La industria alimentaria tiene en la actualidad ofertas que están en el mercado, como 
avance a estos cambios dietarios (productos con ácidos grasos omega-3, 6 o 9, bajos en grasa o con sustitutos de azúcar, entre otros).

Lo anterior es relevante porque podría a futuro aminorar el impacto de un cambio radical en la dieta. Sin embargo, hay que plantear un aviso de alerta porque algunas empresas ofertan dietas personalizadas con base en algunos genes asociados a enfermedades determinadas, pero se percibe que aún faltan estudios concluyentes y derivados de perfiles genéticos particulares, de aquellos sujetos que desean personalizar su alimentación en un binomio sano de dieta-gen.

Finalmente en el rubro educativo es pertinente plantear las siguientes interrogantes ¿quiénes serán los profesionales calificados para atender una consulta de nutrigenética? ¿Cuál será el diseño curricular universitario para cubrir los requisitos de un conocimiento sólido y actualizado sobre esta temática? Lo anterior exige elaborar diseños curriculares para licenciatura, maestría y doctorado, además de abrir espacios para educación continua, es decir educación para toda la vida.

\section{RESUMEN}

El objetivo de este artículo es discutir las perspectivas que ofrece la nutrigenética y su potencial para las recomendaciones nutrimentales individuales. Se establece el papel de los polimorfismos (SNPs) génicos cuya variación incide en la respuesta individual, a diversos patrones dietéticos, lo que resulta la llave maestra en el campo de la nutrigenética. De hecho esta variación genética habrá de considerarse para establecer las recomendaciones nutrimentales de una nutrición personalizada. Este artículo también aborda las perspectivas de la población consumidora frente a esta nueva propuesta (nutrigenética), además de analizar la formación académica de los profesionales de la salud que trabajarán con este nuevo paradigma nutrimental.

Palabras clave: nutrigenética; polimorfismos; dieta personalizada; población consumidora; formación profesional.

Dirigir la correspondencia a:

Profesora

Marta Coronado Herrera

Departamento de Producción Agrícola y Animal

Universidad Autónoma Metropolitana Xochimilco

México

E-mail: nildach@correo.xoc.uam.mx

\section{BIBLIOGRAFÍA}

1. Coronado M. Vega S. Gutiérrez R. Díaz G. Un nuevo paradigma en la salud: Nutrigenética. Nutrición
Clínica 2007; 10:116-25.

2. Coronado M. Vega S. Gutiérrez R. Pérez J. Díaz G. Nichos de investigación, desarrollo e innovación para un conocimiento de vanguardia en el campo de la salud: Nutrigenética. Rev Mexicana Agronegocios 2011; 28:641-58.

3. Martínez E. Genómica nutricional. La nueva nutrición. Nutr Clín Med 2007; I(2): 73-86.

4. Martí A. Moreno Ma. J. Zulet Ma. A. Martínez J. Avances en nutrición molecular: nutrigenómica y/o nutrigenética. Nutr Hospitalaria 2005; 20:157-64.

5. Silveira M. Martínez L. Carraro R. Nutrigenómica, obesidad y salud pública. Rev Española Salud Pública 2007; 81:475-87.

6. Pérez Cruz E. Meléndez Mier G. Zúñiga Rivera A. Genómica nutricional: perspectivas para el futuro. Rev Endocrinol Nutr 2005; 13:190-6.

7. Fernández J. L. Benito J. Panorama actual de la nutrigenómica. ¿Esperanza o Realidad?. Nutr Clín Diet Hospitalaria 2008; 28:38-47.

8. Corella D. Ordovas, J. Single nucleotide polymorphism that influence lipid metabolism: interaction with dietary factors, Ann Rev Nutr 2005; 25:1- 7.

9. Gibney M. Gibney E. Diet, genes and disease: implications for nutrient policy. Proc Nutr Soc 2004; 63: 491-500.

10. Stover P. Nutritional genomics. Physiol Genomics 2004; 16:161-5.

11. Estévez E. Durán C. Genómica nutricional: el estudio de la interacción entre genes y la nutrición humana. Rev Facultad Ciencias Méd (Quito) 2007; 32: 6-13.

12. Simopoulos A. Genetic variation and dietary response: nutrigenetics/nutrigenomics. Asia Pacific J Clin Nutr 2002; 36: S117-S28.

13. Meshkin B. Blum K. Folate nutrigenetics: a convergence of dietary folate metabolism, folic acid supplementation and folate antagonist pharmacogenetics. Drug Metabolism Letters 2007; 1: 55-60.

14. Ascencio C. Torres N. Isoard-Acosta F. GómezPérez F. Hernández-Pando R. Tovar A. Soy protrein affects serum insulina and hepatic SREBP-1 mRNA and reduces fatty liver in rats. J Nutr 2004; 134: 522-9.

15. Tovar A. Manzano N. Torres N. Regulación del colesterol y ácidos grasos en el síndrome nefrótico experimental por las proteínas que se unen a los elementos regulatorios de esteroles (SREBPs): efecto de la soya. Gaceta Méd Mexicana 2005; 141: 27-35.

16. Noriega L. Tovar A. González M. Pando R. Escalante B. Santillán P. Torres N. Pancreatic insulina secretion in rats fed a soy proteín high fat diet depends 
on the interaction between the aminoácido paterrn and isoflavones. J Biol Chem 2007; 282:20657-66.

17. Banz W. Davis J. Peterson R. Iqbal M. Gene expression and adiposity are modified by soy protein in male Zucker diabetic fatty rats. Obesity Res 2004; 12:1907-13.

18. Gómez A. La relación entre la alimentación, la salud y la genómica. Rev OFFARM 2007; 26:1- 8.

19. Almendro V. Gascón P. Nutrigenómica y cáncer. Nutrigenética y Nutrigenómica. Monografías Humanitas 2004; 9:139:52.

20. Brown, L. van der Ouderaa, F. El impacto de la nutrigenómica en la industria alimentaria. Nutrigenética y Nutrigenómica. Monografías Humanitas 2004; 9:121-37.

21. Ronteltap A. van Triyp J. C. M. Renes R. J. Frewer L.J. 2007 Consumer acceptance of technologybased food innovation: lessons for the future of nutrigenomics, Science Direct Appetite 2007; 49: 1-17.

22. Aguilera M. Calleja M. A. Avances moleculares en nutrición y su impacto clínico. Nutr Clín Med 2009; III: 1-19.

23. Palou A. Algunos nuevos retos en nutrición básica y aplicada, Rev Médica Universidad Navarra 2006; 50: 62-70.

24. International Food Information Council, Adaptación de la dieta de acuerdo a sus genes: una búsqueda global, Boletín de noticias Food Insight, Fundación del Consejo Internacional de Información Alimentaria, 2006.

25. Muñoz, E. La nutrigenómica desde la perspectiva del consumidor. Nutrigenética y Nutrigenómica, Monografías Humanitas 2004; 9:71-85.

26. Debusk R. Fogarty C. Ordovas J. Kornman K. Nutritional Genomics in practice: where do we begin?. J Am Diet Assoc 2005; 105: 589-98.

27. Kummer C. Your genomic diet. Technol Rev 2005; $1-6$.

28. Rosen R. Earthman C. Marquart L. Reicks M. Continuing education needs of registered dietitians regarding Nutrigenomics. J Am Diet Assoc 2006; 106:1242-5.

29. Vickery C. Cotugna N. Incorporating human genetics into dietetics curricula remains a challenge. J Am Diet Assoc 2005;105:583-8.

30. Busstra M. Hartog R. Kersten S. Müller M. Design guidelines for the development of digital nutrigenomics learning material for hererogeneous target groups. Advanced Physiol Education 2006; 31:67-75.

31. Guevara M. Tovar A. Larrieta E. Canizales S. Torres $\mathrm{N}$. Increase in HDLC concentration by a dietary portafolio with soy proteína and soluble fiber is associated with the presence of the ABCA1R230C variant in hyperlipidemic Mexican subjects. Molecular Genet Metab 2010; 101:268-72.

32. Ordovas J. M. Integración del medio ambiente y la enfermedad en el análisis "ómico". Rev Española Cardiol 2009; 62:S17-25.

33. Tovar A. Torres N. La nutrigenómica y la nutrigenética. Cuadernos Nutr 2007; 30:177-84.

34. Simopoulos A. Nutrigenetics/Nutrigenomics. Ann Rev Public Health 2010; 31: 53-68. 